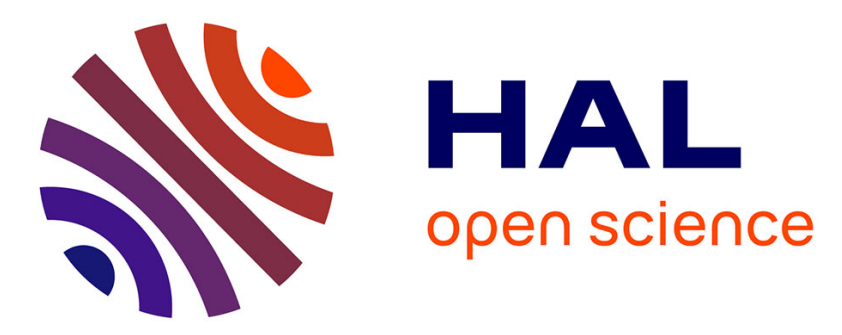

\title{
Des occupations d'altitude du Bronze ancien à la Haille de Pout dans le cirque de Troumouse (Gèdre, Hautes-Pyrénées)
}

\author{
Guillaume Saint-Sever, Maxime Remicourt
}

\section{- To cite this version: \\ Guillaume Saint-Sever, Maxime Remicourt. Des occupations d'altitude du Bronze ancien à la Haille de Pout dans le cirque de Troumouse (Gèdre, Hautes-Pyrénées). Bulletin de la Société préhistorique française, 2017, pp.376-379. 10.3406/bspf.2017.14780 . hal-02322942}

\section{HAL Id: hal-02322942 \\ https://hal.science/hal-02322942}

Submitted on 21 Oct 2019

HAL is a multi-disciplinary open access archive for the deposit and dissemination of scientific research documents, whether they are published or not. The documents may come from teaching and research institutions in France or abroad, or from public or private research centers.
L'archive ouverte pluridisciplinaire HAL, est destinée au dépôt et à la diffusion de documents scientifiques de niveau recherche, publiés ou non, émanant des établissements d'enseignement et de recherche français ou étrangers, des laboratoires publics ou privés. 


\title{
Des occupations d'altitude du Bronze ancien à la Haille de Pout dans le cirque de Troumouse (Gèdre, Hautes-Pyrénées)
}

\author{
Guillaume SAINT-Sever et Maxime RemicourT
}

À la suite de la campagne de sondages conduite par Thomas Perrin et son équipe en 2015 dans le cirque de Troumouse et à la découverte dans le sondage $\mathrm{n}^{\circ} 65$ d'un empierrement de forme oblongue et de tessons attribuables à l'âge du Bronze ancien dans un niveau sous-jacent, ce que devait confirmer ultérieurement une datation radiométrique, une opération de terrain a été entreprise dans le courant de l'année 2016 pour permettre de mieux définir la nature de ces vestiges (funéraires ou domestiques). Cet aménagement est localisé dans le cirque de Troumouse, sur la commune de Gèdre (HautesPyrénées), au lieu-dit la Haille de Pout, en bordure du ravin du Gave de Touyères, sur un replat bordé d'un ruisseau, à 2000 mètres d'altitude.

Malgré une exploration encore partielle du secteur 1, les résultats acquis (Saint-Sever et Remicourt, 2016) montrent que cette zone a été occupée durant plusieurs phases du Bronze ancien et qu'il est pour l'instant possible de discriminer la présence de deux bâtiments successifs, au niveau de l'emprise du sondage $\mathrm{n}^{\circ} 65$. La dernière phase d'occupation (maison 1) est illustrée par un petit bâtiment de forme trapézoïdale $(6 \times 4 \mathrm{~m})$, orienté est-ouest, comportant un mur de pierres sèches à double parement et remplissage interne mixte de terre et petites pierres, d'environ $80 \mathrm{~cm}$ de large. Il est construit avec de gros blocs de granite pouvant atteindre $1 \mathrm{~m}$ de long. Dans la partie interne (partiellement fouillée), nous avons pu identifier deux trous de poteaux et un foyer central contemporains de ce mur. Au sud, un enclos presque circulaire, d'environ 3,5 m de diamètre, est délimité par un cercle de gros blocs de granite qui viennent s'appuyer contre le mur de l'habitation au niveau de la paroi sud (fig. 1).

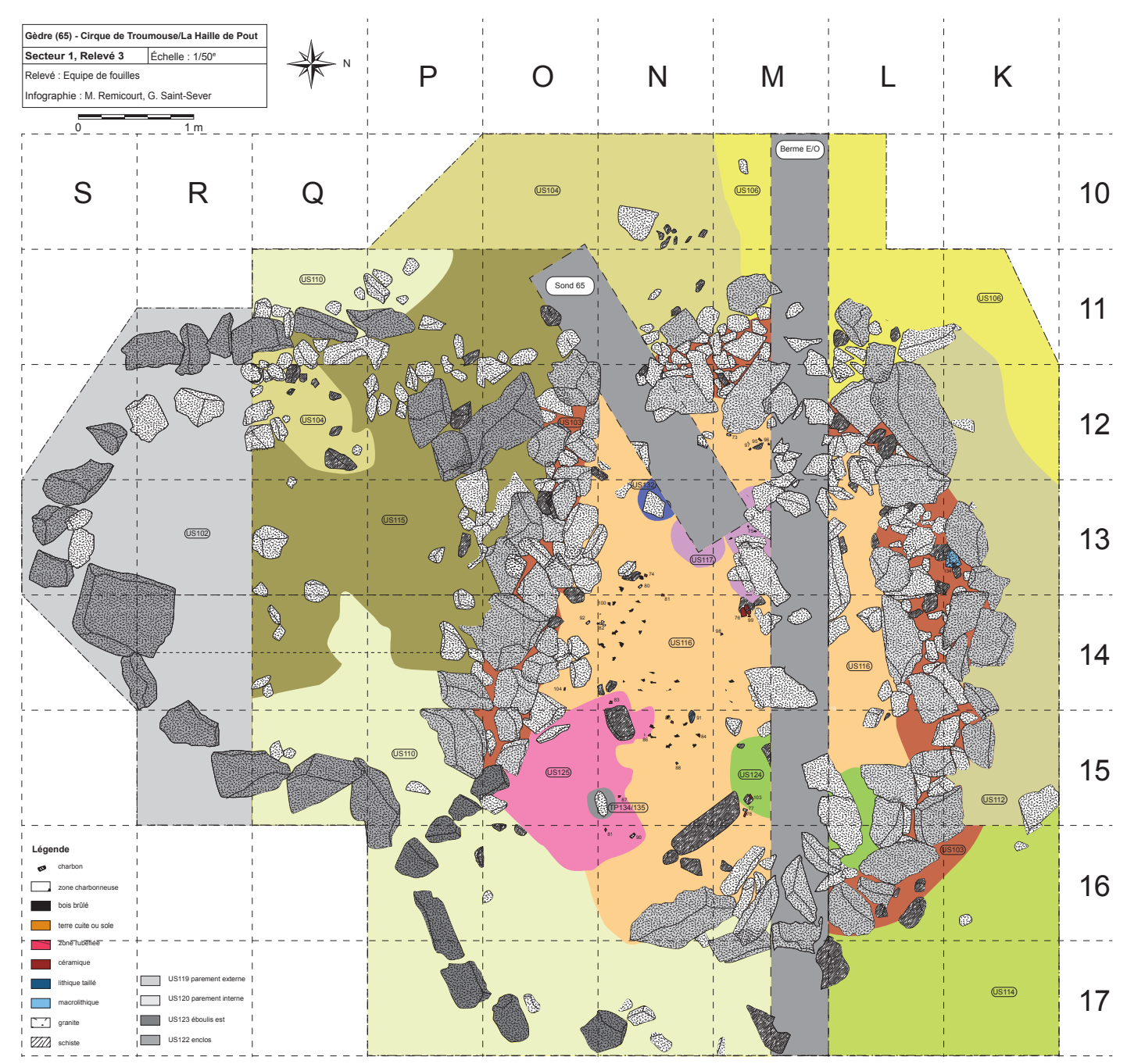

Fig. 1 - Haille de Pout, cirque de Troumouse (Gèdre, Hautes-Pyrénées). Plan de la dernière phase de l'habitat Bronze ancien du secteur 1 (DAO M. Remicourt et G. Saint-Sever). 
Sous les niveaux de destruction de cette première unité domestique, les vestiges d'une seconde habitation ont livré des tessons attribuables au Bronze ancien épi- campaniforme, contemporains des restes céramiques découverts en 2015 (fig. 2). Cette unité se prolonge à la fois au sud et au nord de la première structure en pierres

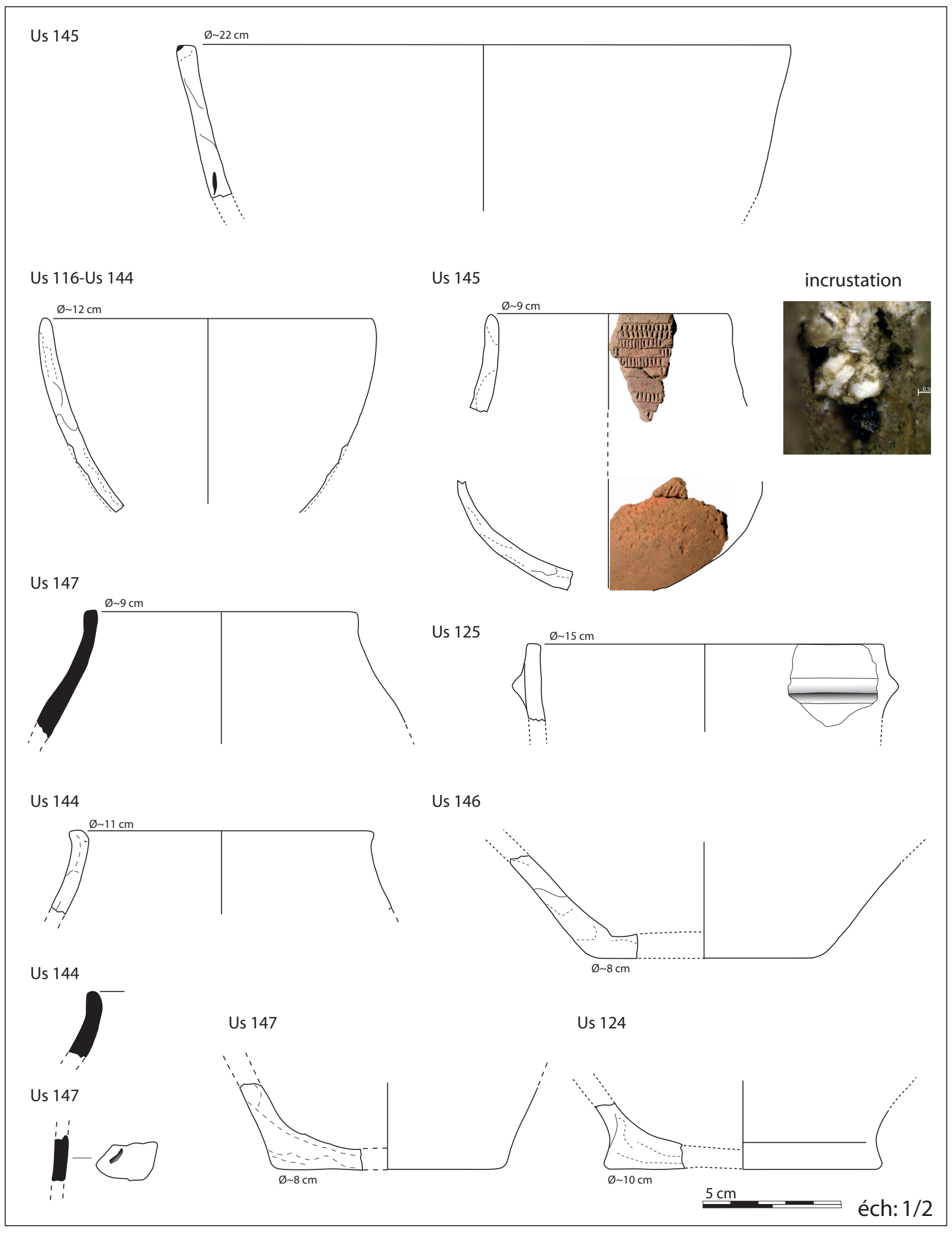

Fig. 2 - Haille de Pout, cirque de Troumouse (Gèdre, Hautes-Pyrénées). Céramiques épicampaniformes découvertes dans les US 124, 144, 145 et 147 du secteur 1 (dessins G. Saint-Sever). 
sèches. Les quelques données disponibles actuellement, montrent qu'il pourrait s'agir d'un bâtiment (maison 2) à base semi-excavée de forme ovalaire ou naviforme, d'une taille d'environ $8 \times 6 \mathrm{~m}$. Cette structure a été incendiée et elle livre dans les niveaux de destructions des restes de planchettes, de brandons et de charbons, ainsi que des céramiques écrasées sur place et des restes lithiques (silex taillés, percuteurs et fragments de meules; fig. $3, \mathrm{n}^{\circ} 2$ ). Cet ensemble a livré la majorité du mobilier archéologique, dont un creuset attestant d'une pratique de la métallurgie du cuivre (fig. $3, n^{\circ} 3$ ). Les indications quant à son mode de construction restent encore ténues, car cette unité n'a été que partiellement fouillée, mais un foyer central a pu être identifié.

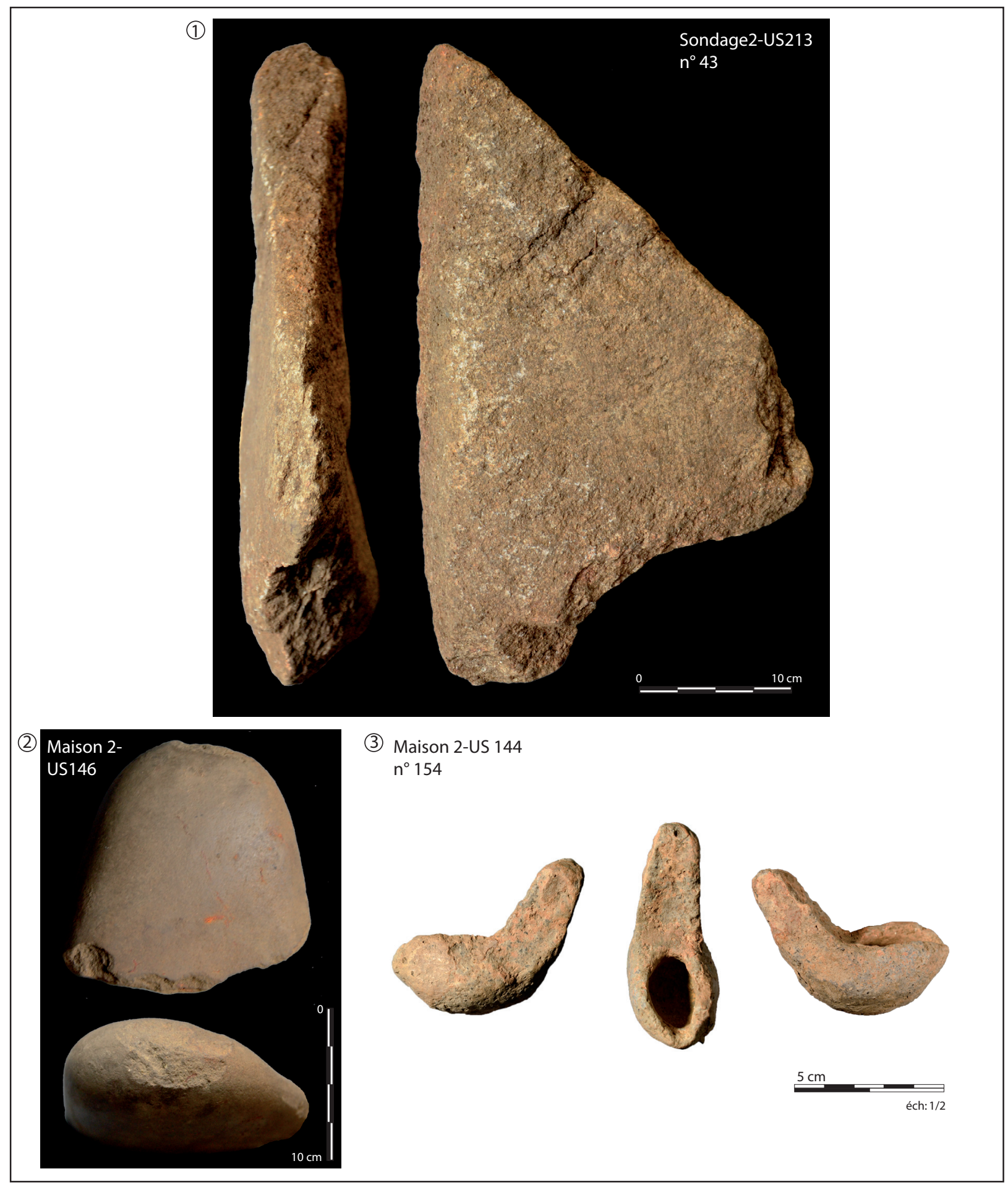

Fig. 3 - Haille de Pout, cirque de Troumouse (Gèdre, Hautes-Pyrénées). Exemple de mobilier macrolithique (meule en granite du Ssondage 2, US 213 et percuteur sur galet-outil du secteur 1, US 146) et d'un creuset-cuillère en argile du secteur 1, US 144 (clichés et DAO M. Remicourt et G. Saint-Sever). 
En complément aux investigations sur le secteur 1 , trois sondages ont été entrepris à proximité de cette zone sur des anomalies comportant des empierrements. Ces derniers ont documenté des témoins plus ou moins importants d'occupations de l'âge du Bronze ancien. Le sondage 2 est le plus riche, puisqu'il a livré les restes de quatre occupations successives sur la terrasse dominant le secteur 1 . On observe la présence d'au moins trois ou quatre bâtiments, qui se réinstallent à la même place, entre des phases d'incendies ou d'abandons. Les premières implantations correspondent à des structures semi-excavées. Tous les niveaux ont livré du mobilier en lien avec les contextes d'habitat, avec des tessons de céramiques attribuables au Bronze ancien, des fragments de meules (fig. 3, $\mathrm{n}^{\circ} 1$ ), des aiguisoirs, des éclats retouchés en silex. Des éléments architecturaux en bois carbonisés, planchettes et brandons, sont également présents. Les deux autres sondages, au nord et au sud du secteur 1, sont moins riches que ce dernier en mobilier archéologique, mais ils ont livré des structures fossoyées à parois rubéfiées, des trous de poteaux et des niveaux de circulation qui ont tous fournis une céramique attribuable au Bronze ancien.

La présence d'un creuset pourrait constituer un des indices pour appréhender la destination de ce type d'implantation et l'exploitation de ce milieu d'altitude. En effet, les environs proches du site sont un réservoir métallifère de pyrites cuivreuses, de galène et de plomb argentifère, en exploitation jusqu'à la fin du XVIII ${ }^{\mathrm{e}}$ siècle. Cette constatation irait dans le sens des données paléoenvironnementales qui indiquent deux pics d'augmentation de l'emprise sur les montagnes pyrénéennes au début du Bronze ancien et au Bronze moyen, en lien avec des pollutions dues à la métallurgie dès le $\mathrm{III}^{\mathrm{e}}$ millénaire avant notre ère (Galop et al., 2001). Ces informations sont corroborées par l'exemple de la mine de cuivre de Causiat, dans la vallée d'Aspe (Urdos, Pyrénées-Atlantiques), où une exploitation de filons de chalcopyrites est identifiée pour la fin du Néolithique et le début de l'âge du Bronze (Kammenthaler et Beyrie, 2007); confirmant ainsi la présence d'activité d'extraction en lien avec la métallurgie pour ces périodes, dans les Pyrénées septentrionales. La proposition d'une occupation de la haute montagne pour des estives pastorales, comme elle est généralement sug- gérée pour ce type d'implantation, n'est toutefois pas pour l'heure à écarter. La poursuite de la fouille et des prospections pédestres pour repérer les filons de chalcopyrites dans ce secteur devraient permettre d'éclaircir ces différents points dans les années à venir.

\section{BIBLIOGRAPHIE}

Galop D., Tual M., Monna F., Domink J., Beyrie A., Marembert F. (2001) - Cinq millénaires de métallurgie en montagne basque. Les apports d'une démarche intégrée alliant palynologie et géochimie isotopique du plomb, SudOuest européen, 11, p. 3-15.

Kammenthaler É., Beyrie A. (2007) - Louvie-Soubiron, Urdos : les origines de l'activité minière et métallurgique dans le haut Béarn, ADLFI. Archéologie de la France Informations. Gallia, http://adlfi.revues.org/7728 [en ligne].

Perrin T. (2015) - PRIMAP 2015. Premiers impacts anthropiques dans les Pyrénées centrales : approches multi-proxy (archéologie, paléoenvironnement). Prospection-inventaire avec sondages à Gèdre (Hautes-Pyrénées), rapport de fouille programmée, service régional de l'Archéologie de Midi-Pyrénées, Toulouse, $158 \mathrm{p}$.

Saint-Sever G., Remicourt M. (2016) - Fouille archéologique de la Haille de Pout, cirque de Troumouse, Gèdre (HautesPyrénées), rapport de fouille programmée, service régional de l'Archéologie de Midi-Pyrénées, Toulouse, 119 p.

\section{Guillaume SAINT-Sever} docteur associé,

UMR 5608, TRACES « Rhadamante »

Maxime Remicourt membre associé, UMR 5608, TRACES «PRBM »

université de Toulouse, maison de la Recherche, 5, allées Antonio-Machado, F-31058 Toulouse cedex 9 